\title{
Design of Transverse Spinning of Light with Globally Unique Handedness
}

\author{
Xianji Piao, Sunkyu Yu, and Namkyoo Park ${ }^{*}$ \\ Photonic Systems Laboratory, Department of Electrical and Computer Engineering, \\ Seoul National University, Seoul 08826, Korea
}

(Received 4 January 2018; published 15 May 2018)

\begin{abstract}
Access to the transverse spin of light has unlocked new regimes in topological photonics. To achieve the transverse spin from nonzero longitudinal fields, various platforms that derive transversely confined waves based on focusing, interference, or evanescent waves have been suggested. Nonetheless, because of the transverse confinement inherently accompanying sign reversal of the field derivative, the resulting transverse spin handedness of each field experiences spatial inversion, which leads to a mismatch between the intensities of the field and its spin component and hinders the global observation of the transverse spin. Here, we reveal a globally pure transverse spin of the electric field in which the field intensity signifies the spin distribution. Starting from the target spin mode for the inverse design of required spatial profiles of anisotropic permittivities, we show that the elliptic-hyperbolic transition around the epsilon-near-zero permittivity allows for the global conservation of transverse spin handedness of the electric field across the topological interface between anisotropic metamaterials. Extending to the non-Hermitian regime, we develop annihilated transverse spin modes to cover the entire Poincaré sphere of the meridional plane. This result realizes the complete optical analogy of three-dimensional quantum spin states.
\end{abstract}

DOI: 10.1103/PhysRevLett.120.203901

The very nature of transverse waves of light has hindered our access to the rich physics and applications related to longitudinal fields, e.g., the optical counterpart of threedimensional quantum spin states. However, the recent emergence of plasmonics [1] and metamaterials [2] that provide superior light confinement has lifted the restriction on longitudinal fields, leading to unconventional dynamics such as left-handed metamaterials [3], artificial magnetics [4], full-field reconstruction [5], and plasmonic radiation [6]. By controlling the phase of the longitudinal field relative to the transverse component, the hidden quantity of transverse spin ( $T$ spin) of electromagnetic fields $[7,8]$ has also been realized. Intensive studies were subsequently conducted to examine the optical $T$ spin and offer unexplored degrees of freedom for 3D light-matter interactions [9-12] especially for topological photonics $[9,11]$.

Although the platforms for optical $T$ spin can be classified according to the excitation method of nonzero longitudinal components of light using either a propagating [13-15] or an evanescent wave [9,16,17], the necessary condition for both cases is identical: the transverse confinement of light that drives nonzero longitudinal fields. Nonetheless, compared with the longitudinal spin (or

Published by the American Physical Society under the terms of the Creative Commons Attribution 4.0 International license. Further distribution of this work must maintain attribution to the author(s) and the published article's title, journal citation, and DOI. circular polarization of light), which can be defined as a globally pure and exclusive quantity, the $T$ spin usually remains a local quantity due to the spatial inversion of its handedness $[7,8,10,13-16,18]$ [opposite sign of spin across $x=0$ in Figs. 1(a)-1(d)], which leads to a mismatch between the field intensity $|\boldsymbol{\Psi}|^{2}$ and each spin intensity component, $\left|\psi_{+}\right|^{2}$ or $\left|\psi_{-}\right|^{2}$. Considering the current absence of $T$-spin filters that are analogous to circular polarizers for the longitudinal spin, the realization of a globally pure $T$-spin mode that achieves $|\Psi|^{2}=\left|\psi_{+}\right|^{2}$ is strongly desired. Such a $T$-spin mode will allow for the utilization of the field intensity $|\boldsymbol{\Psi}|^{2}$ as the direct measuring quantity of the $T$ spin $\psi_{+}$, in the context of far-field transport and $T$-spin mode observations.

In this Letter, we propose a realization of pure $T$-spin modes for the electric field that preserve unique handedness in a global domain [same sign of spin across $x=0$ in Fig. 1(e)]. Noticing that the spatial inversion of $T$ spin originates from the transverse light confinement, we reveal that the globally unique handedness of $T$ spin can be achieved by implementing a topological transition around the uniaxial epsilon-near-zero (ENZ) permittivity in the transverse coordinate. By inversely designing the landscapes of anisotropic permittivities according to the target spin wave profile, we demonstrate the construction of arbitrary profiles of $T$-spin modes [Figs. 1(f)-1(h)] including zero spin [Fig. 1(i)], which preserves the global spin purity. Our results achieve complete coverage of the Poincaré sphere including the longitudinal component, 


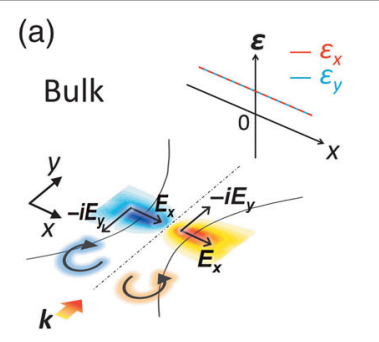

(c) Spin intensity
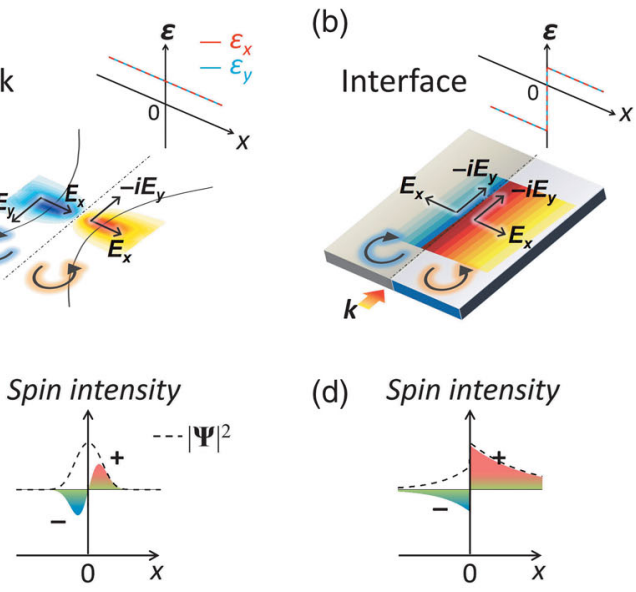

(d) Spin intensity

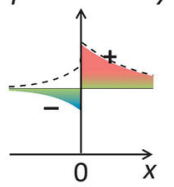

(e)
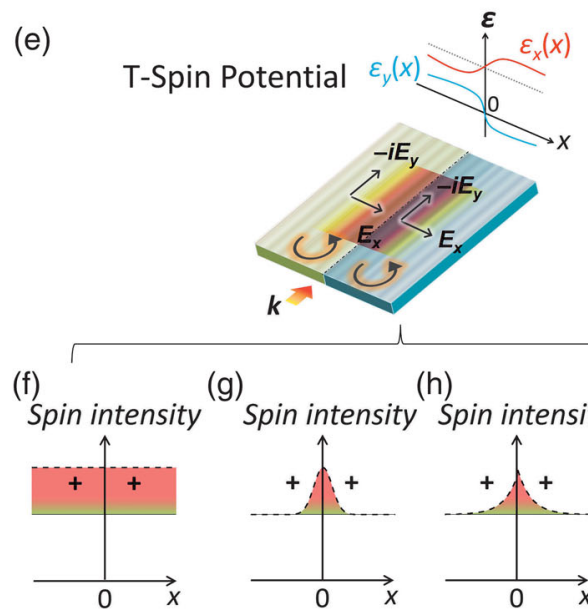

(g)

(h)

(i)
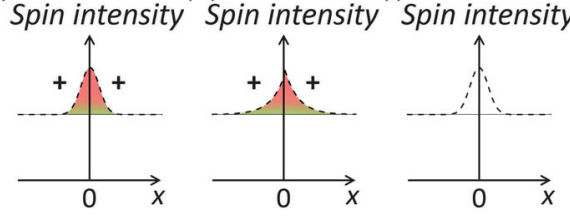

FIG. 1. Illustration of a globally pure $T$-spin mode. (a)-(d) Conventional $T$-spin realizations from (a) a fundamental Gaussian beam in a homogeneous isotropic medium $(\varepsilon>0$ for all $x)$ and from (b) an evanescent plasmonic wave at an interface between metal and dielectric media $[\varepsilon(x<0)<0$ and $\varepsilon(x>0)>0]$. (e)-(i) Proposed $T$-spin potential between two inhomogeneous anisotropic media around the uniaxial ENZ. The overlapping fields in (a), (b), and (e) indicate each spin intensity distribution. (c),(d), (f)-(i) Corresponding net spin intensity profiles $\left|\psi_{+}(x)\right|^{2}-$ $\left|\psi_{-}(x)\right|^{2}$ compared to the entire field intensity $|\Psi(x)|^{2}$ (dashed lines). (c) Gaussian and (d) plasmonic waves. (f)-(h) $T$-spin waves with globally pure handedness $\left[|\boldsymbol{\Psi}(x)|^{2}=\left|\psi_{+}\right|^{2}\right]$. (f) Plane, (g) Gaussian, and (h) evanescent wave. (i) A $T$-spin-annihilated wave without handedness $\left[\left|\psi_{+}(x)\right|^{2}=\left|\psi_{-}(x)\right|^{2}\right]$.

thereby paving the way toward full access to the 3D polarization states of propagating light.

We focus on the transverse magnetic (TM, magnetic field $H_{z}$ along the $z$ axis) mode in the $x-y$ plane, and it can be readily extended to the transverse electric (TE) mode with dual symmetry [19]. Conventional $T$-spin realizations in isotropic media are derived from the confinement of light by exploiting waves focused in bulk media [Fig. 1(a) for a fundamental Gaussian beam] or evanescent waves at the interface [Fig. 1(b) for a plasmonic wave]. In Figs. 1(a) and 1(b), the longitudinal component of the displacement vector $\left(D_{y}=i \cdot \partial_{x} H_{z} / \omega\right)$ accompanies the spatial sign inversion along the transverse axis $x$ to achieve the confinement $\left[\partial_{x} H_{z}(x<0)>0\right.$ and $\partial_{x} H_{z}(x>0)<0$ ]. Because $D_{x}$ is continuous, the sign inversion of $D_{y}$ leads to the inevitable sign inversion of the $T$ spin along the transverse axis regardless of prior realization methods that utilize isotropic media [Figs. 1(a) and 1(b)], thus resulting in $|\Psi|^{2} \neq\left|\psi_{+}\right|^{2}$ [Figs. 1(c) and 1(d)]. We note that this spatial inversion of the relative phase between $D_{x}$ and $D_{y}$ in Fig. 1 always occurs in any lossless confined TM waves (Note S1 in Ref. [20]) and in vectorial confined waves (Note S2 in Ref. [20] for the $T$-spin inversion in vectorial higher-order Hermite-Gaussian beams [22]).

The aforementioned sign reversals of the $\mathbf{D}$ field and the resulting spin handedness of the $\mathbf{E}$ field can be overcome via the constitutive relation $\mathbf{E}=\boldsymbol{\varepsilon}^{-1} \mathbf{D}$. To release the restriction on the $\mathbf{D}$ field, we consider anisotropic materials of $\boldsymbol{\varepsilon}(x)$, which adds new degrees of freedom to the control of the electric field irrespective of the displacement field. For example, with $\varepsilon_{y}(x<0)>0$ and $\varepsilon_{y}(x>0)<0$, the globally unique handedness of the spin [Fig. 1(e)] could be achieved, where its profile could also be further controlled by $\varepsilon_{x}(x)$ [Figs. 1(f)-1(i)].

In the context of the inverse design of optical potentials from the target eigenmode, the $T$-spin mode of globally "pure" handedness in the entire space $\left[\psi_{-}(x)=0\right]$ can be molded. We assume electrically anisotropic materials having one-dimensional variations of $\varepsilon_{x, y}(x)$ and a nonmagnetic feature $\left(\mu=\mu_{0}\right)$. The electric field has the form of $\mathbf{E}(x, y)=\boldsymbol{\Psi}(x) e^{-i \beta y}$, where $\boldsymbol{\Psi}(x)$ is the field envelope and $\beta$ is the $y$-propagating wave vector. In the $T$-spin representation of $\boldsymbol{\Psi}(x)=\psi_{+}(x) \mathbf{e}_{+}+\psi_{-}(x) \mathbf{e}_{-}$for the $z$-axis spins $\mathbf{e}_{ \pm}=\left(\mathbf{e}_{\mathbf{x}} \pm i \mathbf{e}_{\mathbf{y}}\right) / 2^{1 / 2}$ "transverse" to $y$-propagating waves, a globally positive $T$-spin mode $\boldsymbol{\Psi}(x)=\psi_{+}(x) \mathbf{e}_{+}$ from $\psi_{-}(x)=0$ is achieved with the anisotropic and inhomogeneous optical potential (Note S3 in Ref. [20] for derivation)

$$
\begin{aligned}
& \varepsilon_{x}(x)=\varepsilon_{e}+\frac{\sqrt{\varepsilon_{e}}}{k_{0}} \partial_{x}\left(\log \psi_{+}\right), \\
& \varepsilon_{y}(x)=-\frac{1}{k_{0}^{2}} \frac{\partial_{x}^{2} \psi_{+}}{\psi_{+}}-\frac{\sqrt{\varepsilon_{e}}}{k_{0}} \partial_{x}\left(\log \psi_{+}\right),
\end{aligned}
$$

where $k_{0}=2 \pi / \lambda_{0}$ is the free-space wave number and $\varepsilon_{e}$ is the effective permittivity of the $+y$-propagating $(\beta>0) T$ spin eigenmode from $\beta^{2}=\varepsilon_{e} k_{0}^{2}$. Because $\mathbf{e}_{ \pm}$represents the transverse spinning of the electric field $\mathbf{E}(x, y)$, the spin follows the Abraham momentum expression [31], signifying the classical momentum of electromagnetic fields [32]. Note that the $T$-spin handedness of the eigenmode possesses the directionality, which corresponds to the spin-momentum locking [9] (Note S4 in Ref. [20] for directionality and mirror symmetry dependencies). Equation (1) can be applied to obtain potential landscapes for any nodeless spatial profiles of the target $\psi_{+}(x)$. 
First, we consider the trivial case of a $T$-spin "plane wave," where $\partial_{x} \psi_{+}=0$. The required potential profile then becomes constant as $\varepsilon_{x}(x)=\varepsilon_{e}$ and $\varepsilon_{y}(x)=0$. This solution reveals that zero longitudinal permittivity $\varepsilon_{y}=0$, which corresponds to uniaxial ENZ [33-35] materials, imposes the full degree of freedom on the longitudinal field $E_{y}$ and thus allows the emergence of the ideal $T$-spin plane wave $\left(D_{y}=\varepsilon_{y} E_{y}=0\right)$. However, from $\varepsilon_{y}(x)=0$, any $E_{y}(x)$ is able to satisfy Maxwell's equations, hindering the exclusive excitation of the pure $T$ spin. We thus explore the condition of nontrivial "confined" $T$-spin eigenmodes distinct from the trivial plane wave case.

Without loss of generality, we consider an example of the Gaussian profile $\left[\psi_{+}(x)=\exp \left[-x^{2} /\left(2 \sigma^{2}\right)\right]\right]$ while keeping $\psi_{-}(x)=0$ [Fig. 2(a), $\sigma=5 \lambda_{0}$ ]. The required optical potential [Fig. 2(b)] is then "inhomogeneous" anisotropic (a)

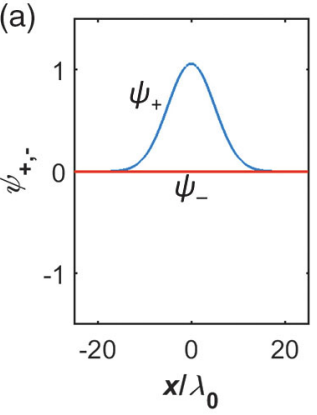

(c)

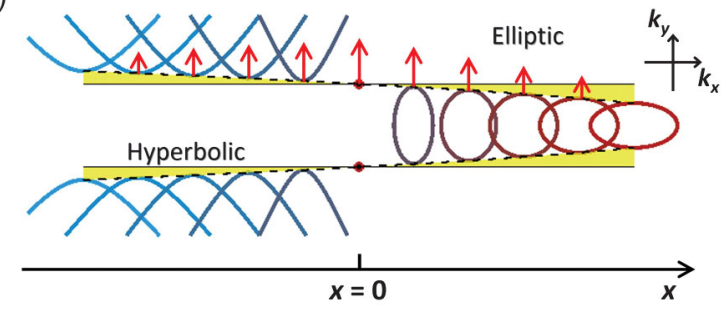

(d)

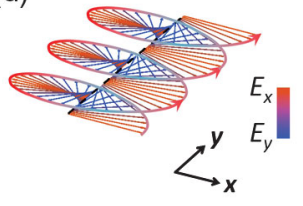

(b)

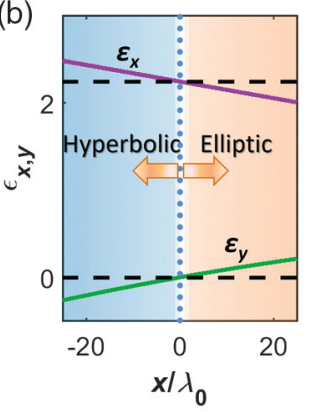

media (Note S5 in Ref. [20] for field profiles of the target eigenmode with different modal sizes). Remarkably, although $\varepsilon_{x}(x)$ has its distribution near the target effective permittivity $\varepsilon_{e}$ [upper black dashed line in Fig. 2(b)], $\varepsilon_{y}$ exhibits an intriguing transition from negative to positive values near $x=0$, thus realizing the topological transition [36] of the isofrequency contour (IFC) between hyperbolic [26] and elliptic materials [Fig. 2(c)]. Compared with the plane wave solution of constants $\varepsilon_{x}(x)=\varepsilon_{e}$ and $\varepsilon_{y}(x)=0$, the spatial transition of the IFC near uniaxial ENZ $\left[\varepsilon_{y}(x<0)>0\right.$ and $\left.\varepsilon_{y}(x>0)<0\right]$ not only allows the pure $T$-spin handedness of the target eigenmode [Fig. 2(d)] but also yields a momentum mismatch [yellow regions in Fig. 2(c)], which leads to the confinement of the eigenmode near $x \sim 0$ [Fig. 2(e)]. We also note that the target $T$-spin eigenmode is separated from the other eigenmodes in both the spatial and momentum domains (Note S6 in Ref. [20] for nearby eigenmodes), which enables the exclusive excitation of the $T$ spin without the other polarization states.

Although the result in Fig. 2 demonstrates the existence of a confined $T$-spin mode with a globally "pure" handedness, the spatially varying condition of anisotropic materials may hinder practical implementations. Because the modal profile of $e^{-\alpha x}$ allows for "homogeneous" realizations [Eq. (1) of $\varepsilon_{x}(x)=\varepsilon_{e}$ and $\varepsilon_{y}(x)=-\alpha^{2} / k_{0}^{2}$, we explore the condition of potential landscapes for the confined modal profiles $\psi_{+}(x)=\exp \left(-|x|^{g} / 2 \sigma^{g}\right)$ [Figs. 3(a) and 3(b), $g=2$ to 1 , from Gaussian to exponential]. As expected, the potential becomes homogeneous for $g \sim 1$ except for the discontinuity in $\partial_{x} \psi_{+}$at $x=0$ and the corresponding singularity of $\varepsilon_{y}(x)$. By neglecting the contribution of the second-order derivative $\partial_{x}^{2} \psi_{+}$in Eq. (1) for the ansatz function $\psi_{+}(x) \sim e^{-\alpha|x|}$, Eq. (1) can be used to derive the topological-transition interface between hyperbolic $\left[\varepsilon_{x}, \varepsilon_{y}\right]=\left[\varepsilon_{e}+\right.$ $\Delta \varepsilon,-\Delta \varepsilon]$ and elliptic $\left[\varepsilon_{x}, \varepsilon_{y}\right]=\left[\varepsilon_{e}-\Delta \varepsilon, \Delta \varepsilon\right]$ materials [ $\Delta \varepsilon=\left(\varepsilon_{e}^{1 / 2} / k_{0}\right) \alpha>0$, Fig. 3(c); see Note S7 in Ref. [20] for the interface mode derivation], where the value of $\Delta \varepsilon$ determines the confinement of the eigenmode $\psi_{+}(x) \sim e^{-\alpha|x|}$.

Figure 3(d) represents the electric field distribution of the $T$-spin interface mode for the material parameters $\varepsilon_{e}=2$ and $\Delta \varepsilon=0.5$. As expected, the signs of the transverse electric field $E_{x}$ and the following net spin are preserved along the entire $x$ axis, achieving $\left|\psi_{+}\right| \gg\left|\psi_{-}\right|$[Fig. 3(e)]. Moreover, the $\Delta \varepsilon$-dependent discontinuity in the transverse field $E_{x}\left[\psi_{x}\right.$ in Fig. 3(d)] indicates that a negligible excitation of $\psi_{-}$occurs except in the case of deepsubwavelength confinements (Note S8 in Ref. [20]). We also note that the stability and purity of the $T$-spin interface mode are fairly tolerant to the perturbation in material parameters (Note S9 in Ref. [20]).

Figures 3(f) and 3(g) show the excitation of the $T$-spin mode $\psi_{+}$and suppression of $\psi_{-}$at the interface that employs the platform of hyperbolic [26] and elliptic layered 

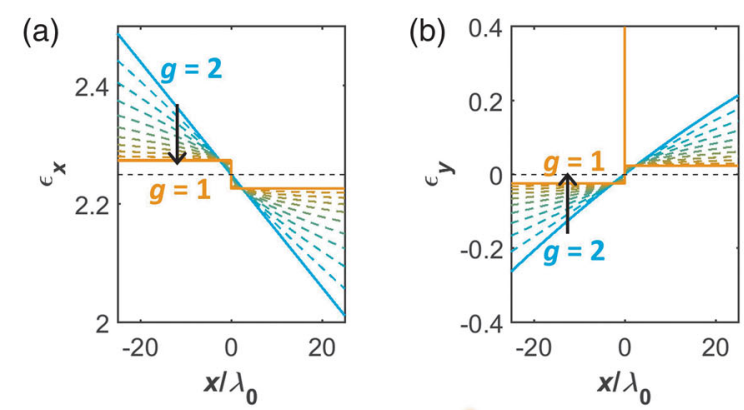

(c)
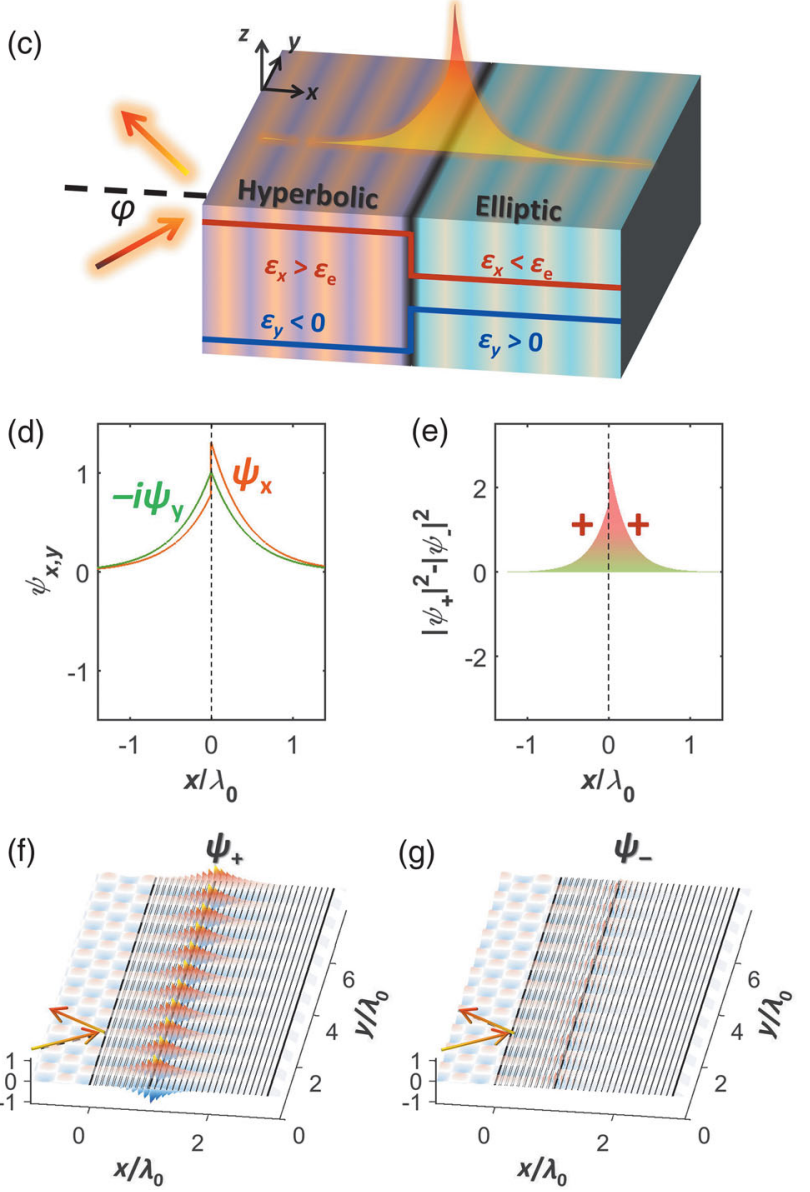

FIG. 3. $T$-spin mode using homogeneous materials. (a) $\varepsilon_{x}$ and (b) $\varepsilon_{y}$ for the target $T$ spin $\psi_{+}(x)=\exp \left(-|x|^{g} / 2 \sigma^{g}\right)$. (c) Schematic for the $T$-spin interface mode using homogeneous media of hyperbolic and elliptic IFC. Colored arrows represent the incident and reflected waves for the excitation ( $\varphi$ incident angle). (d) Field amplitude $\psi_{x, y}$ and (e) net spin intensity $\left|\psi_{+}\right|^{2}-$ $\left|\psi_{-}\right|^{2}$ of the $T$-spin interface mode. (f),(g) Scattering-matrixcalculated [30] $T$-spin excitations $\left(\psi_{+}, \psi_{-}\right)$in hyperbolic and elliptic metamaterials $\left(\varphi=43.2^{\circ}\right) .\left(\varepsilon_{x}, \varepsilon_{y}\right)=(2.5,-0.5)$ at $x<0$ and $\left(\varepsilon_{x}, \varepsilon_{y}\right)=(1.5,0.5)$ at $x \geq 0$ for $\varepsilon_{e}=2$ and $\Delta \varepsilon=0.5$.

metamaterials (Note S10 in Ref. [20] for detailed parameters). The $\psi_{+}$-dominant $T$-spin mode is excited with an oblique plane wave incidence, satisfying the phase-matching condition same as in the case of the prism coupling for plasmon excitations [29] (Note S11 for excitation conditions in Ref. [20]). To detect the $T$-spin mode, transverse
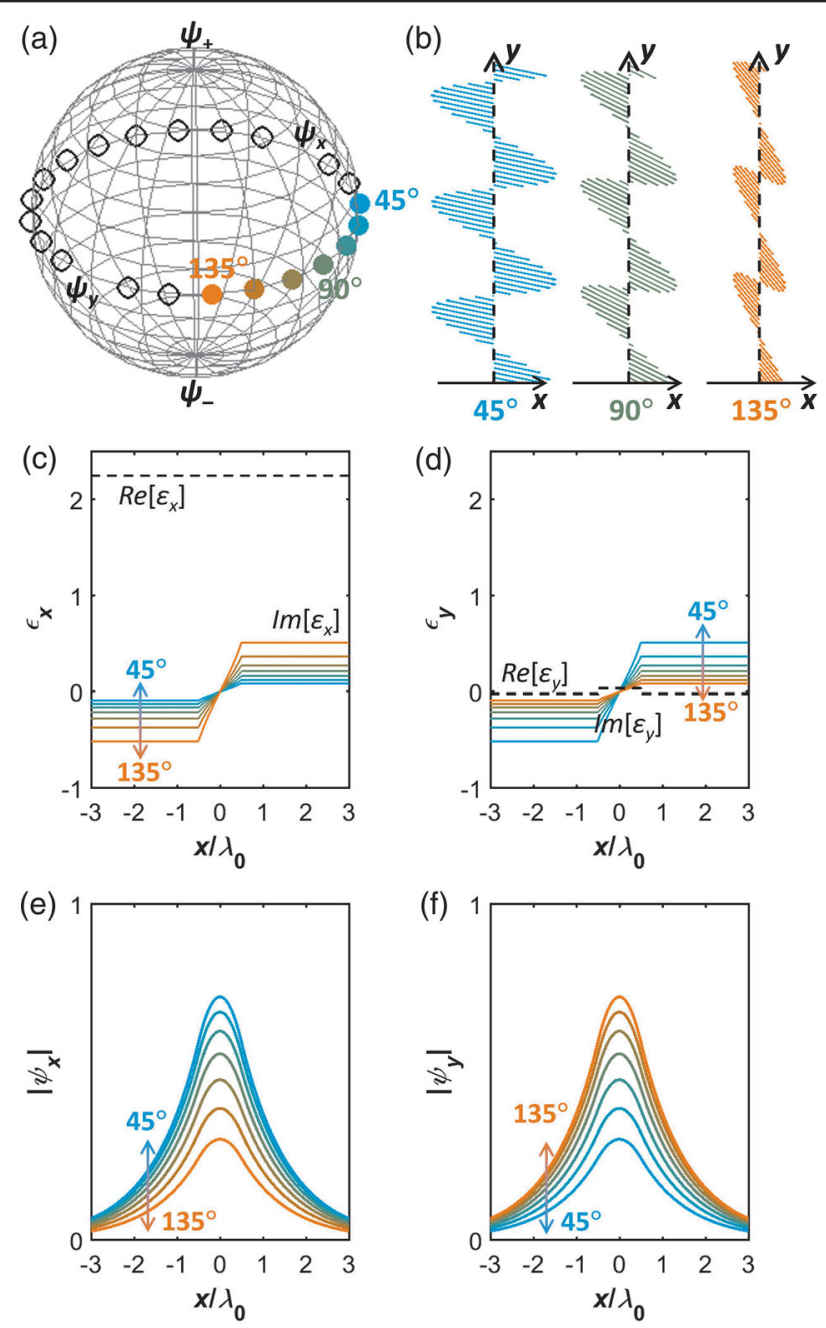

FIG. 4. Annihilation of $T$ spin in complex potentials. (a) Representations of zero $T$-spin modes on the $T$-spin Poincaré sphere. The angles indicate the phase difference between positive and negative $T$ spins $\psi_{-} / \psi_{+}=e^{i \theta}$. (b) Polarization evolution during the propagation for each $\theta$. (c), (d) Designed complex anisotropic permittivities (c) $\varepsilon_{x}$ and (d) $\varepsilon_{y}$ for the target modes of each $\theta$. Dotted lines indicate the real part, and solid lines represent the imaginary part. (e) $\left|\psi_{x}\right|$ and (f) $\left|\psi_{y}\right|$ components in the eigenmodes of each $\theta . \varepsilon_{e}=2.25$.

$\left(E_{x}\right)$ and longitudinal $\left(E_{y}\right)$ fields have to be measured with the same boundary condition. This type of measurement can be achieved by the vector-field microscope [37], scanning the electric field over the $x-y$ plane. The homogeneous realization in Fig. 3 also opens up the possibility of $T$-spin mode excitation at the interface between natural anisotropic media [38,39].

Extending the realization of pure $T$-spin waves further, we challenge the feasibility of obtaining a fundamental but unexplored state of polarization (SOP): the zero- $T$-spin mode, on the Poincaré sphere of the meridional plane [Fig. 4(a)], which we call the " $T$-spin Poincaré" sphere. This zero- $T$-spin mode corresponds to a linear polarization "oblique" to the propagation direction [Fig. 4(b)]. In 
isotropic Hermitian media, the emergence of a longitudinal field with an intrinsic $\pi / 2$ phase difference with respect to the transverse field always accompanies the $T$-spin component. To annihilate this $T$ spin, the $T$-spin modes with different signs should be degenerate with the same amplitude $\left(\psi_{-}=\psi_{+} e^{i \theta}\right)$, where $\theta$ denotes the polarization angle with respect to the propagation direction. As shown in Eq. (S3) in Ref. [20], the degeneracy of two $T$-spin modes $\psi_{ \pm}$with a phase difference $\theta$ is achieved with the complex anisotropic permittivity $\varepsilon_{x, y}$. Figures 4(c) and 4(d) show the required landscapes of complex potentials $\varepsilon_{x, y}$, for the zero$T$-spin modes of different polarization angles $\theta$ [Figs. 4(e) and 4(f)]. Almost all SOPs on the $T$-spin Poincare sphere can be covered with spatially varying complex anisotropic media except for diverging states of $\theta=0^{\circ}$ (purely transversal wave) and $\theta=180^{\circ}$ (purely longitudinal wave). This $T$-spin Poincaré sphere together with the classical Poincare sphere completes the access to the 3D polarization and spin eigenmodes with full degrees of freedom. Note that the "annihilation" of the $T$ spin in complex potentials originating from the $\pi / 2$ phase difference between transverse and longitudinal fields is distinct from the "creation" of the longitudinal spin in parity-timesymmetric potentials [40].

We demonstrated the realization of pure $T$-spin modes without the spatial inversion of the handedness, by applying the topological transition around the uniaxial ENZ. In the proposed structure, the field intensity itself becomes the measuring quantity of the $T$ spin. With the zero transverse spin that derives in-phase oscillations between transverse and longitudinal fields, all SOPs on the $T$-spin Poincaré sphere can be accessed. The full access to the $T$-spin Poincaré sphere will be the foundation of the applications of 3D polarizations. This new SOP together with conventional ones enables the complete representation of 3D spin and their superposition, which allows for multifaceted degrees of freedom for topological photonics [41] and spin-orbit coupling [11]. An unconventional mode at the topological interface in $k$ space [36] also inspires its 3D extension using isofrequency "surfaces" with different topology.

We also note that our result using the Abraham expression leads to an intriguing situation: the apparent contradiction between Abraham $\left(\mathbf{E} \times \mathbf{H} / c^{2}\right)$ and Minkowski $(\mathbf{D} \times \mathbf{B})$ momenta. In the proposed $T$-spin mode, the fields of $\mathbf{E}, \mathbf{H}$, and $\mathbf{B}$ vectors and the $D_{x}$ field are nodeless functions, while only the $D_{y}$ field has a single node with the sign inversion at $x=0$. This result shows the globally unique handedness of the Abraham $T$ spin in contrast to the spatial inversion of the Minkowski $T$-spin handedness. Such an inconsistency that has not been observed in the conventional $T$ spin in isotropic materials [9,13-17] will give an insight into the separate control of "the Abraham classical momentum" and "the Minkowski pseudomomentum".
The authors thank F. Nori for valuable advice and discussion on the $T$-spin mode. We acknowledge financial support from the National Research Foundation of Korea (NRF) through the Global Frontier Program (2014M3A6B3063708), the Korea Research Fellowship Program (2016H1D3A1938069), and the Basic Science Research Program (2016R1A6A3A04009723), all funded by the Korean government.

*nkpark@snu.ac.kr

[1] S. A. Maier, Plasmonics: Fundamentals and Applications (Springer Science \& Business Media, New York, 2007).

[2] N. Engheta and R. W. Ziolkowski, Metamaterials: Physics and Engineering Explorations (John Wiley \& Sons, New York, 2006).

[3] R. Marqués, F. Medina, and R. Rafii-El-Idrissi, Role of bianisotropy in negative permeability and left-handed metamaterials, Phys. Rev. B 65, 144440 (2002).

[4] F. Shafiei, F. Monticone, K. Q. Le, X.-X. Liu, T. Hartsfield, A. Alù, and X. Li, A subwavelength plasmonic metamolecule exhibiting magnetic-based optical Fano resonance, Nat. Nanotechnol. 8, 95 (2013).

[5] T. Bauer, S. Orlov, U. Peschel, P. Banzer, and G. Leuchs, Nanointerferometric amplitude and phase reconstruction of tightly focused vector beams, Nat. Photonics 8, 23 (2014).

[6] L. J. Wong, I. Kaminer, O. Ilic, J. D. Joannopoulos, and M. Soljačić, Towards graphene plasmon-based free-electron infrared to x-ray sources, Nat. Photonics 10, 46 (2016).

[7] A. Aiello, P. Banzer, M. Neugebauer, and G. Leuchs, From transverse angular momentum to photonic wheels, Nat. Photonics 9, 789 (2015).

[8] K. Y. Bliokh and F. Nori, Transverse and longitudinal angular momenta of light, Phys. Rep. 592, 1 (2015).

[9] K. Y. Bliokh, D. Smirnova, and F. Nori, Quantum spin Hall effect of light, Science 348, 1448 (2015).

[10] M. Antognozzi, C. Bermingham, R. Harniman, S. Simpson, J. Senior, R. Hayward, H. Hoerber, M. Dennis, A. Bekshaev, K. Bliokh, and F. Nori, Direct measurements of the extraordinary optical momentum and transverse spindependent force using a nano-cantilever, Nat. Phys. 12, 731 (2016).

[11] K. Y. Bliokh, F. Rodríguez-Fortuño, F. Nori, and A. V. Zayats, Spin-orbit interactions of light, Nat. Photonics 9, 796 (2015).

[12] A. Canaguier-Durand, A. Cuche, C. Genet, and T. W. Ebbesen, Force and torque on an electric dipole by spinning light fields, Phys. Rev. A 88, 033831 (2013).

[13] M. Neugebauer, T. Bauer, P. Banzer, and G. Leuchs, Polarization tailored light driven directional optical nanobeacon, Nano Lett. 14, 2546 (2014).

[14] T. Bauer, M. Neugebauer, G. Leuchs, and P. Banzer, Optical Polarization Möbius Strips and Points of Purely Transverse Spin Density, Phys. Rev. Lett. 117, 013601 (2016).

[15] M. Neugebauer, T. Bauer, A. Aiello, and P. Banzer, Measuring the Transverse Spin Density of Light, Phys. Rev. Lett. 114, 063901 (2015). 
[16] K. Y. Bliokh, A. Y. Bekshaev, and F. Nori, Extraordinary momentum and spin in evanescent waves, Nat. Commun. 5, 3300 (2014).

[17] K. Y. Bliokh and F. Nori, Transverse spin of a surface polariton, Phys. Rev. A 85, 061801 (2012).

[18] A. Y. Bekshaev, K. Y. Bliokh, and F. Nori, Transverse Spin and Momentum in Two-Wave Interference, Phys. Rev. X 5, 011039 (2015).

[19] K. Y. Bliokh, A. Y. Bekshaev, and F. Nori, Dual electromagnetism: Helicity, spin, momentum and angular momentum, New J. Phys. 15, 033026 (2013).

[20] See Supplemental Material at http://link.aps.org/ supplemental/10.1103/PhysRevLett.120.203901 for (S1) relative phase inversion of $D$ fields, (S2) $T$-spin inversion in Hermite-Gaussian beams, (S3) optical potentials for $T$-spin modes, (S4) propagation directions and mirror symmetry, (S5) modal size dependency, (S6) nearby eigenmodes, (S7) derivation of $T$-spin interface mode, (S8) trade-off between confinement and handedness, (S9) tolerance of $T$-spin interface mode, (S10) metamaterial $T$-spin interface, and (S11) $T$-spin excitation conditions, which includes Refs. [21-30].

[21] M. Berry, Five momenta, Eur. J. Phys. 34, 1337 (2013).

[22] K. Duan, B. Wang, and B. Lü, Propagation of HermiteGaussian and Laguerre-Gaussian beams beyond the paraxial approximation, J. Opt. Soc. Am. A 22, 1976 (2005).

[23] S. Longhi, Quantum-optical analogies using photonic structures, Laser Photonics Rev. 3, 243 (2009).

[24] R. F. Oulton, V. J. Sorger, D. Genov, D. Pile, and X. Zhang, A hybrid plasmonic waveguide for subwavelength confinement and long-range propagation, Nat. Photonics 2, 496 (2008).

[25] T. C. Choy, Effective Medium Theory: Principles and Applications (Oxford University Press, New York, 2015), Vol. 165.

[26] A. Poddubny, I. Iorsh, P. Belov, and Y. Kivshar, Hyperbolic metamaterials, Nat. Photonics 7, 948 (2013).

[27] M. S. Kumar, X. Piao, S. Koo, S. Yu, and N. Park, Out of plane mode conversion and manipulation of surface plasmon polariton waves, Opt. Express 18, 8800 (2010).

[28] N. Janunts, K. Baghdasaryan, K. V. Nerkararyan, and B. Hecht, Excitation and superfocusing of surface plasmon polaritons on a silver-coated optical fiber tip, Opt. Commun. 253, 118 (2005).
[29] J. Sambles, G. Bradbery, and F. Yang, Optical excitation of surface plasmons: An introduction, Contemp. Phys. 32, 173 (1991).

[30] S. G. Tikhodeev, A. L. Yablonskii, E. A. Muljarov, N. A. Gippius, and T. Ishihara, Quasiguided modes and optical properties of photonic crystal slabs, Phys. Rev. B 66, 045102 (2002).

[31] J. P. Gordon, Radiation forces and momenta in dielectric media, Phys. Rev. A 8, 14 (1973).

[32] R. N. Pfeifer, T. A. Nieminen, N. R. Heckenberg, and H. Rubinsztein-Dunlop, Colloquium: Momentum of an electromagnetic wave in dielectric media, Rev. Mod. Phys. 79, 1197 (2007).

[33] A. Alù, M. G. Silveirinha, A. Salandrino, and N. Engheta, Epsilon-near-zero metamaterials and electromagnetic sources: Tailoring the radiation phase pattern, Phys. Rev. B 75, 155410 (2007).

[34] L. Alekseyev, E. Narimanov, T. Tumkur, H. Li, Y. A. Barnakov, and M. Noginov, Uniaxial epsilon-near-zero metamaterial for angular filtering and polarization control, Appl. Phys. Lett. 97, 131107 (2010).

[35] I. Liberal and N. Engheta, Near-zero refractive index photonics, Nat. Photonics 11, 149 (2017).

[36] H. N. Krishnamoorthy, Z. Jacob, E. Narimanov, I. Kretzschmar, and V. M. Menon, Topological transitions in metamaterials, Science 336, 205 (2012).

[37] K. Lee, H. Kihm, J. Kihm, W. Choi, H. Kim, C. Ropers, D. Park, Y. Yoon, S. Choi, D. Woo, J. Kim, B. Lee, Q. Park, C. Lienau, and D. Kim, Vector field microscopic imaging of light, Nat. Photonics 1, 53 (2007).

[38] M. W. McCall, I. J. Hodgkinson, and Q. Wu, Birefringent Thin Films and Polarizing Elements (World Scientific, Singapore, 2014).

[39] L. V. Alekseyev, V. A. Podolskiy, and E. E. Narimanov, Homogeneous hyperbolic systems for terahertz and farinfrared frequencies, Adv. OptoElectron. 2012, 267564 (2012).

[40] S. Yu, H. S. Park, X. Piao, B. Min, and N. Park, Lowdimensional optical chirality in complex potentials, Optica 3, 1025 (2016).

[41] L. Lu, J. D. Joannopoulos, and M. Soljačić, Topological photonics, Nat. Photonics 8, 821 (2014). 